\title{
GENERAL RADIATION FORMULAE FOR A RELATIVISTIC CHARGED PARTICLE MOVING IN CURVED MAGNETIC FIELD LINES: THE SYNCHROCURVATURE RADIATION MECHANISM
}

\author{
K. S. CHENG \\ Department of Physics, University of Hong Kong \\ AND \\ J. L. ZHANG \\ Center of Astrophysics, University of Science and Technology of China, People's Republic of China, and \\ Department of Physics, University of Hong Kong \\ Received 1995 May 30; accepted 1995 November 14
}

\begin{abstract}
We calculate the radiation spectrum, characteristic frequency, and power emitted by a relativistic charged particle moving in curved magnetic field lines with arbitrary pitch angle, which can be approximated by a curved spiral trajectory. We obtain some universal formulae which can be identical to either synchrotron radiation or curvature radiation in certain parametric regions, but in general the radiation features of this new mechanism can significantly differ from those of these two known mechanisms. Since magnetic field lines are generally curved in many real systems (e.g., a pulsar magnetosphere), this new mechanism can more realistically describe the radiation from relativistic charged particles in these systems. We also find that the degree of polarization is the best quantity to differentiate this new mechanism from the other two mechanisms.
\end{abstract}

Subject headings: polarization — radiation mechanisms: nonthermal — relativity

\section{INTRODUCTION}

Among all the radiation mechanisms in pulsars, synchrotron and curvature radiations are the most important (for a general review of pulsar radiation mechanisms seee Michel 1991 and Mészáros 1992). In previous discussions, they have been treated as two independent mechanisms. The synchrotron radiation formula is derived assuming a uniform and straight magnetic field, while the formula for curvature radation assumes that the charged particle moves along a curved field line with similar results for the characteristic frequency, radiation, power, and frequency distribution, except that the cyclotron radius is replaced by the radius of curvature. In the magnetosphere of a pulsar charged particles are moving in curved magnetic lines. Their trajectories are likely to be spiral curves along the curved field lines. It is obvious that the radiation from charged particles with this type of trajectory cannot be described by either the simple synchrotron radiation or the curvature radiation theories. In calculating the radio emission from Jupiter, many authors (Chang \& Davis 1962; Field 1961; Korchak 1962; Thorne 1963; Ortwein, Chang, \& Davis 1966) have considered radiation from charged particles moving in dipolar field lines. However, they still used the synchrotron radiation formula for charged particles moving in uniform and straight magnetic field lines, which may or may not be correct. Nevertheless, justification for using this formula seems necessary. We want to remark that radiation from charged particles in this kind of trajectory is still synchrotron radiation in origin, like that of curvature radiation, but its radiation properties have obvious differences from those of simple synchrotron radiation. In order to differentiate between synchrotron radiation from charged particles in straight field lines and that from charged particles in curved field lines, we call the former simple synchrotron radiation and the latter synchro-curvature radiation. In this paper, we will deal with the following questions. What is the general formula to describe the radiation from charged particles with this type of motion, namely, a curved spiral motion? Under what conditions are simple synchrotron radiation and curvature radiation still valid? What are the implications of this new mechanism?

For simplicity, we will not consider the real shape of the magnetic field, e.g., dipolar field, etc. Instead, we will assume a rather simple situation: a circular magnetic field. Furthermore, the guiding center is assumed to be moving along the magnetic field lines. Under these approximations, particles will exhibit a circular motion around the guiding center, which is moving with a constant speed along the magnetic field lines. Of course, we have ignored the decrease in velocity caused by the radiation. We need to emphasize again that the magnetic field and particle trajectory are only an approximation of the real situation. This circular field approximation should be valid as long as the radius of the curvature of the magnetic field does not change significantly over a distance roughly equal to $c T_{\text {cyc }}$, where $T_{\text {cyc }}$ is the cyclotron period.

\section{EQUATION OF MOTION}

Let us consider a charged particle with mass $m$ and charge $e$ that is moving within a circular magnetic field. In the $(x, y)$ coordinate plane, we use the polar coordinate $\operatorname{system}(\rho, \varphi)$. The equation for the magnetic field can be expressed as

$$
\boldsymbol{B}=B_{0} \varphi^{0}=B_{0}\left(\cos \varphi \boldsymbol{j}^{0}-\sin \varphi \boldsymbol{i}^{0}\right),
$$

where $B_{0}$ is assumed to be a constant for the convenience of later quantitative estimation. The velocity of the guiding center in 
this type of field structure can be expressed as

$$
v_{G}=\left(v_{\varphi}-\frac{\gamma m v_{z} v_{\varphi} c}{e B_{0} \rho}\right) \boldsymbol{e}_{\varphi}^{0}+\frac{\gamma m v_{\varphi}^{2} c}{e B_{0} \rho} e_{z}^{0} .
$$

This shows that the guiding center can only have drift velocity transverse to the magnetic field line in the $z$-direction. In the $\rho$-direction, the drift velocity is zero. In one cyclotron period, $v_{z}$ alternates direction and thus $\left\langle v_{\varphi} v_{z}\right\rangle=0$. Therefore, in the $\varphi^{0}$-direction, the guiding center will move with nearly uniform velocity $v_{\varphi}=v \cos \alpha$. The drift velocity in the $z$-direction is given by

$$
\left(v_{G}\right)_{z}=\frac{\gamma m v_{\varphi}^{2} c}{e B_{0} \rho}=\frac{v^{2} \cos ^{2} \alpha}{\omega_{B} \rho} .
$$

where $\alpha$ is the angle between $\boldsymbol{B}$ and $v$ and $\omega_{B}=e B_{0} /(\gamma m c)$. We can see that the particle will drift with a uniform velocity $\left(v_{G}\right)_{z}$ along the $z$-direction. As the magnetic field, $\rho$, and $\alpha$ increase, the drift velocity decreases. For example, in the outer magnetosphere of a young pulsar, where the typical values of the parameters are $B=10^{6} \mathrm{G}, \gamma=10^{7}, v \sim c$, and $\rho \sim 10^{8} \mathrm{~cm}$, $\left(v_{G}\right)_{z} \leq 10^{6} \mathrm{~cm} \mathrm{~s}^{-1} \ll c$. Such a velocity will only allow the guiding center to drift away a distance less than $10^{4} \mathrm{~cm}$ or cause a fractional change in magnetic field less than $10^{-4}$. In the main text, we will not consider this drift velocity when we are calculating the frequency distribution. Also, we will assume that the particle exhibits circular motion around the guiding center, which is moving along the magnetic field lines with a uniform velocity. We want to emphasize that the drift velocity of the guiding center does not change our general results; it causes a minor modification of the characteristic frequency, spectrum, and total radiation power loss, but the forms of these quantities remain unchanged (for details see Appendix C).

In order to describe circular motion of a particle within the magnetic field lines, we choose a coordinate system $O^{\prime}, X^{\prime}, Y^{\prime}, Z^{\prime}$ whose center coincides with the guiding center of the particle. Let $\hat{\boldsymbol{x}}^{\prime}$ coincide with $\boldsymbol{\rho}^{\mathbf{0}}$ and $\hat{\boldsymbol{y}}^{\prime}$ coincide with $\varphi^{\mathbf{0}}$, then $\hat{\boldsymbol{z}}^{\prime}$ coincides with $z^{0}$. The particle will rotate within the magnetic field lines on the $\left(X^{\prime}, Z^{\prime}\right)$-plane. Let $r_{B}=c \sin \alpha / \omega_{B}$ be its cyclotron radius; then

$$
\boldsymbol{r}=r_{B} \cos \omega_{B} t \rho^{0}+r_{B} \sin \omega_{B} t z^{0} .
$$

The motion of the guiding center along the magnetic field line can be described by the variation of $\rho^{0}$ and $\varphi^{0}$. Suppose we assume that initially, when $t=0$, the guiding center is on the $y$-axis, and let $\Omega_{0}$ be its angular velocity. Obviously,

$$
\begin{aligned}
& \rho^{0}=-\sin \Omega_{0} t \boldsymbol{i}^{0}+\cos \Omega_{0} t \boldsymbol{j}^{0}, \\
& \varphi^{0}=-\cos \Omega_{0} t \boldsymbol{i}^{0}-\sin \Omega_{0} t \boldsymbol{j}^{0},
\end{aligned}
$$

where $\Omega_{0}=v_{\varphi} / \rho$. Hence the position vector of the particle at a particular instant can be expressed as

$$
\begin{aligned}
\boldsymbol{r}= & \boldsymbol{\rho}+\boldsymbol{r}^{\prime}=-\left\{\rho \sin \Omega_{0} t+\frac{r_{B}}{2}\left[\sin \left(\Omega_{0}+\omega_{B}\right) t+\sin \left(\Omega_{0}-\omega_{B}\right) t\right]\right\} \boldsymbol{i}^{0} \\
& +\left\{\rho \cos \Omega_{0} t+\frac{r_{B}}{2}\left[\cos \left(\Omega_{0}-\omega_{B}\right) t+\cos \left(\Omega_{0}+\omega_{B}\right) t\right]\right\} j^{0}+r_{B} \sin \omega_{B} t \boldsymbol{k}^{0} .
\end{aligned}
$$

\section{RADIATION SPECTRUM}

In order to simplify the calculation of the spectrum, which is measured by a distant observer, we will assume that the magnetic field lines are uniform in the length scale $\sim 2 r_{c}^{*} / \gamma$, in which the light pulse is formed. Here $r_{c}^{*}$ is the effective radius of curvature, which will be defined later. Without loss of generality, we can assume that the observer is located on the $(X, Z)$ plane. Also we let $\theta_{0}$ be the angle between the position vector of the observer and $\left(-x^{0}\right)$. Therefore, the unit position vector pointing to the observer is given by

$$
n=-\cos \theta_{0} i^{0}+\sin \theta_{0} k^{0} .
$$

The spectral energy density for an arbitrary accelerated particle in the range of solid angles between $\Omega_{0}$ and $\Omega_{0}+d \Omega_{0}$, and in the frequency range between $\omega$ and $\omega+d \omega$ is given by (Peratt 1991)

$$
\frac{d^{2} E}{d \omega d \Omega}+\frac{e^{2} \omega^{2}}{4 \pi^{2} c}\left|\int_{-T / 2}^{T / 2} e^{i \omega[t-(n \cdot r / c)}[n \times(n \times \beta)] d t\right|^{2} .
$$

In order to obtain the observed spectrum, we need to calculate the above integral. After using some common approximations for calculating the synchrotron radiation (for details see Appendix A), we obtain

$$
\frac{d^{2} E_{\perp}}{d \omega d \Omega}=\frac{e^{2} \omega^{2} A_{\perp}^{2}}{4 \pi^{2} c}\left|\int_{-\infty}^{\infty} \tau \exp \left[i f\left(Q_{1}^{2} \tau+\frac{1}{3} \tau^{3}\right)\right] d \tau\right|^{2}=\frac{e^{2} \omega^{2} A_{\perp}^{2} Q_{1}^{4}}{3 \pi^{2} c} K_{2 / 3}^{2}\left(\frac{2}{3} f\right)
$$

and

$$
\frac{d^{2} E_{\|}}{d \omega d \Omega}=\frac{e^{2} \omega^{2} A_{\|}^{2}}{4 \pi^{2} c}\left|\int_{-\infty}^{\infty} \exp \left[i f\left(Q_{1}^{2} \tau+\frac{1}{3} \tau^{3}\right)\right] d \tau\right|^{2}=\frac{e^{2} \omega^{2} A_{\|}^{2} Q_{1}^{2}}{3 \pi^{2} c} K_{1 / 3}^{2}\left(\frac{2}{3} f\right),
$$


where

$$
\begin{gathered}
Q_{1}^{2} \equiv 1+2 \gamma^{2}\left[2 \sin ^{2}\left(\frac{\alpha-\theta_{0}}{2}\right)-\frac{r_{B}}{\rho} \cos \alpha \cos \theta_{0}\right] \\
Q_{2}^{2} \equiv\left(\frac{r_{B}^{2}+\rho r_{B}-3 \rho^{2}}{\rho^{3}} \cos ^{3} \alpha \cos \theta_{0}+\frac{3}{\rho} \cos \alpha \cos \theta_{0}+\frac{1}{r_{B}} \sin ^{3} \alpha \sin \theta_{0}\right) \frac{1}{r_{B}}, \\
f \equiv \frac{1}{2} \frac{\omega}{\gamma^{3} Q_{2} c}, \\
A_{\perp}^{2} \equiv\left[\left(\rho+r_{B}\right) \Omega_{0}^{2}+r_{B} \omega_{B}^{2}\right]^{2} \frac{1}{c^{6} Q_{2}^{4} \gamma^{4}}, \\
A_{\|}^{2} \equiv\left[\left(\rho+r_{B}\right) \Omega_{0}^{2} \sin \theta_{0}-r_{B} \omega_{B} \cos \theta_{0}\right]^{2} \frac{1}{c^{2} Q_{2}^{2} \gamma^{2}} .
\end{gathered}
$$

Before we calculate the average radiation power spectrum, we need to point out that $\theta_{0}$ is practically very close to $\alpha$. This is because the phase factor in equations (3.3) and (3.5) is related to $Q_{1}^{2}$, which has a large value unless $\left\{2 \sin ^{2}\left[\left(\alpha-\theta_{0}\right) / 2\right]\right.$ $\left.-\left(r_{B} / \rho\right) \cos \alpha \cos \theta_{0}\right\} \rightarrow 0$. Otherwise, the phase factor oscillates rapidly and makes the positive and negative shifts cancel each other. Furthermore, we will show that the angle $\chi$ between $n$ and $v$ satisfies $\cos \chi=1-\left\{2 \sin ^{2}\left[\left(\alpha-\theta_{0} / 2\right]-\left(r_{B} / \rho\right) \cos \alpha\right.\right.$ $\left.\cos \theta_{0}\right\}$. Therefore, the dominant contribution of the integral in equation (3.2) is when $\cos \chi \approx 1$, which implies $\alpha \approx \theta_{0}$ or $Q_{1} \approx 1$. In other words, the radiation is concentrated within a narrow cone centered in the direction of motion, and the characteristic frequency can be estimated by $(4 / 3) f=\omega / \omega_{c}$ or

$$
\omega_{c}=\frac{3}{2} \gamma^{3} c \frac{1}{\rho}\left[\frac{\left(r_{B}^{3}+\rho r_{B}^{2}-3 r_{B} \rho^{2}\right)}{\rho r_{B}^{2}} \cos ^{4} \alpha+\frac{3 \rho}{r_{B}} \cos ^{2} \alpha+\frac{\rho^{2}}{r_{B}^{2}} \sin ^{4} \alpha\right]^{1 / 2} .
$$

We will have the following limiting cases:

1. When $\rho=\infty, \alpha \neq 0$, the characteristic frequency becomes the characteristic frequency of synchrotron radiation, namely,

$$
\omega_{c}=\frac{3}{2} \gamma^{3} c \frac{\sin ^{2} \alpha}{r_{B}}=\frac{3}{2} \gamma^{3} c \frac{\sin ^{2} \alpha}{c \sin \alpha / \omega_{B}}=\frac{3}{2} \gamma^{3} \omega_{B} \sin \alpha .
$$

2. When $\alpha=0$, so $r_{B}=0$, the situation is similar to the curvature radiation. In this case,

$$
\omega_{c}=\frac{3}{2} \gamma^{3} c \frac{1}{\rho}\left(1-\frac{3 \rho}{r_{B}}+\frac{3 \rho}{r_{B}}\right)^{1 / 2}=\frac{3}{2} \gamma^{3} \frac{c}{\rho} .
$$

To calculate the distribution of the radiation power, we will first show that $\left\{2 \sin ^{2}\left[\left(\alpha+\theta_{0}\right) / 2\right]-\left(r_{B} / \rho\right) \cos \alpha \cos \theta_{0}\right\}$ is related to the inclination angle $\chi$ between $\boldsymbol{v}$ and $\boldsymbol{n}$ when $t \approx 0$. We have

$$
\boldsymbol{v}=-r_{B} \omega_{B} \sin \omega_{B} t \boldsymbol{\rho}^{0}+\left(\rho+r_{B} \cos \omega_{B} t\right) \Omega_{0} \varphi^{0}+r_{B} \omega_{B} \cos \omega_{B} t \boldsymbol{k}^{0}
$$

and

$$
\boldsymbol{n}=\cos \theta_{0} \sin \Omega_{0} t \boldsymbol{\rho}^{0}+\cos \theta_{0} \cos \Omega_{0} t \boldsymbol{\varphi}^{0}+\sin \theta_{0} \boldsymbol{k}^{0} .
$$

Therefore $\chi$ must satisfy the following relation:

$$
v \cos \chi=\boldsymbol{n} \cdot \boldsymbol{v}=r_{B} \omega_{B} \cos \theta_{0} \sin \omega_{B} t \sin \Omega_{0} t+\left(\rho+r_{B} \cos \omega_{B} t\right) \Omega_{0} \cos \theta_{0} \cos \Omega_{0} t+r_{B} \omega_{B} \cos \omega_{B} t \sin \theta_{0} .
$$

Using $r_{B}=(v \sin 0) / \omega_{B}$ and $\rho=(v \cos \alpha) / \Omega_{0}$, we obtain

$$
\cos \chi=\sin \alpha \cos \theta_{0} \sin \omega_{B} t \sin \Omega_{0} t+\cos \alpha \cos \theta_{0} \cos \Omega_{0} t+\frac{\Omega_{0}}{\omega_{B}} \sin \alpha \cos \theta_{0} \cos \omega_{B} t \cos \Omega_{0} t+\sin \alpha \cos \omega_{B} t \sin \theta_{0} .
$$

Initially, $\chi$ clearly satisfies

$$
\cos \chi=1-\left[2 \sin ^{2}\left(\frac{\alpha-\theta_{0}}{2}\right)-\frac{r_{B}}{\rho} \cos \alpha \cos \theta_{0}\right] .
$$

We have argued that the main contribution to the radiation power appears when $\left\{2 \sin ^{2}\left[\left(\alpha-\theta_{c}\right) / 2\right]-\left(r_{B} / \rho\right) \cos \alpha\right.$ $\left.\cos \theta_{0}\right\} \rightarrow 0$, which means that the velocity is pointing toward the observer, namely, $\chi \approx 0$, and within the range of $\Delta \chi \sim 1 / \gamma$. Therefore,

$$
Q_{1}^{2}=\left\{1+2 \gamma^{2}\left[2 \sin ^{2}\left(\frac{\alpha-\theta_{0}}{2}\right)-\frac{r_{B}}{\rho} \cos \alpha \cos \theta_{0}\right]\right\} \approx 1+\gamma^{2} \chi^{2}
$$


On the other hand, from equation (3.15) we can approximate

$$
\begin{aligned}
& \cos \chi \approx \frac{1}{c}\left[\left(r_{B}+\rho\right) \Omega_{0} \cos \theta_{0}+r_{B} \omega_{B} \sin \theta_{0}\right] \\
& \sin \chi \approx \frac{1}{c}\left[\left(r_{B}+\rho\right) \Omega_{0} \sin \theta_{0}-r_{B} \omega_{B} \cos \theta_{0}\right] .
\end{aligned}
$$

Therefore we can rewrite equation (A14) as

$$
\{\boldsymbol{n} \times(\boldsymbol{n} \times \boldsymbol{v})\}_{\boldsymbol{x}^{\prime \prime}} \approx c \chi
$$

Therefore equation (A18) becomes

$$
\frac{d^{2} E_{\|}}{d \omega d \Omega}=\frac{e^{2} \omega^{2}}{4 \pi^{2} c^{3} Q_{2}^{2} \gamma^{4}}\left|\int_{-\infty}^{\infty} \gamma \chi \exp \left\{i f\left[\left(1+\gamma^{2} \chi^{2}\right) r+\frac{1}{3} \tau^{3}\right]\right\} d \tau\right|^{2},
$$

and equation (A16) becomes

$$
\frac{d^{2} E_{\perp}}{d \omega d \Omega}=\frac{e^{2} \omega^{2}\left[\left(r_{B}+\rho\right) \Omega_{0}^{2}+r_{B} \omega_{B}^{2}\right]^{2}}{4 \pi^{2} c^{7} Q_{2}^{4} \gamma^{4}}\left|\int_{-\infty}^{\infty} \tau \exp \left\{i f\left[\left(1+\gamma^{2} \chi^{2}\right) \tau+\frac{1}{3} \tau^{3}\right]\right\} d \tau\right|^{2} .
$$

Since equations (3.22) and (3.23) contain the radiation angle $\chi$ between $\boldsymbol{n}$ and $\boldsymbol{v}$ explicitly, after integrating over the radiation angle, we will find $d P_{\perp} / d \omega$ and $d P_{\|} / d \omega$. Strictly speaking, when we integrate the radiation angle, we should consider the functional relationship between $Q_{2}$ and $\chi$. But we have shown that the radiation is concentrated in the $\chi \simeq 0$ direction. Therefore, we can replace $\theta_{0}$ by $\alpha$ in the expression of $Q_{2}$, and the error caused will not be significant.

To find the radiation power per unit frequency and per unit solid angle, we should divide the spectral energy density (eq. [3.23]) by the pulse emitting time between two successive observations (i.e., the period of motion). For the synchrotron radiation, this time interval is its rotation period $T=2 \pi / \omega_{B}$. For the case when the magnetic field is curved, the time interval between two pulses is not equal to $T$. We will denote it by $T^{*}=2 \pi / \omega^{*}$. Meanwhile, in the original flat magnetic field, the radiation within a period should concentrate in a layer with an angular width $1 / \gamma$ of a cone with an opening angle $\alpha$. For the integrals about the solid angle $d \Omega$, we need only consider the contribution in this layer, i.e., $d \Omega=2 \pi$ sin $\alpha d \chi$. For the case when the magnetic field is curved, the opening angle of the cone will no longer be $\alpha$. We will denote it by $\alpha^{*}$. We will determine $\alpha^{*}$ and $\omega^{*}$ later. From equations (3.22) and (3.23), we can obtain the spectra of the radiation power:

$$
\left.\left|\frac{d P_{\perp}}{d \omega}=\frac{e^{2} \omega^{2}\left[\left(r_{B}+\rho\right) \Omega_{0}^{2}+r_{B} \omega_{B}^{2}\right]^{2}}{4 \pi^{2} c^{7} Q_{2}^{4} \gamma^{5}} \omega^{*} \sin \alpha^{*} \int_{-\infty}^{\infty} d \bar{\chi}\right| \int_{-\infty}^{\infty} \tau \exp \left\{i f\left[\left(1+\bar{\chi}^{2}\right) \tau+\frac{1}{3} \tau^{3}\right]\right\} d \tau\right|^{2}
$$

and

$$
\frac{d P_{\|}}{d \omega}=\frac{e^{2} \omega^{2} \omega^{*} \sin \alpha^{*}}{4 \pi^{2} c^{3} Q_{2}^{2} \gamma^{5}} \int_{-\infty}^{\infty} \bar{\chi}^{2} \mid\left.\int_{-\infty}^{\infty} \tau \exp \left\{\text { if }\left[\left(1+\bar{\chi}^{2}\right) \tau+\frac{1}{2} \tau^{3}\right]\right\} d \tau\right|^{2} d \bar{\chi},
$$

where $\bar{\chi}=\gamma \chi$. Here we have extended the integration range of $\bar{\chi}$ to $\pm \infty$ (because $\gamma \gg 1$ ).

Due to the Doppler shift, the spectra measured by an observer are not given by equations (3.24) and (3.25), which are the emission spectra. Instead, we should replace $T^{*}$ by $T^{\prime}$, which is given by

$$
T^{\prime}=\left(1-\frac{v}{c} \cos \alpha \cos \theta^{\prime}\right) \frac{2 \pi}{\omega^{*}} \approx\left(1-\frac{v}{c} \cos ^{2} \alpha\right) \frac{2 \pi}{\omega^{*}} \approx\left(\frac{1}{2 \gamma^{2}}+\sin ^{2} \alpha\right) \frac{2 \pi}{\omega^{*}} .
$$

However, we wish to point out that the above factor only occurs for the case of a single particle. An ensemble of particles will smear out the above factor.

In order to calculate the emission spectrum given in equations (3.24) and (3.25), we need to express $\omega^{*} \sin \alpha^{*}$ in an explicit form. In the case of straight magnetic field lines, $\omega_{B} \sin \alpha=c / r_{c}$, where $r_{c}$ is the radius of curvature of the particle trajectory. Since the radiation properties are solely determined by the radius of curvature and velocity (Ochelkov \& Usov 1980), we can identify $\omega^{*} \sin \alpha^{*}=c / r_{c}^{*}$, where $r_{c}^{*}$ is the instantaneous radius of curvature of the particle trajectory evaluated at the emission point and can be expressed as

$$
\frac{1}{r_{c}^{*}}=\left|\frac{\dot{\boldsymbol{r}} \times \ddot{\boldsymbol{r}}}{|\dot{\boldsymbol{r}}|^{3}}\right|
$$

From equation (3.13) we obtain

$$
r_{c}^{*} \approx \frac{c^{2}}{\left[r_{B} \omega_{B}^{2}+\left(\rho+r_{B}\right) \Omega^{2}\right]}
$$


where we have used $|\dot{\boldsymbol{r}}| \approx c$. Therefore, equations (3.24) and (3.25) can be written as

$$
\begin{aligned}
& \frac{d P_{\perp}}{d \omega}=\frac{e^{2} \omega^{2}\left[\left(r_{B}+\rho\right) \Omega_{0}^{2}+r_{B} \omega_{B}^{2}\right]^{3}}{4 \pi^{2} c^{9} Q_{2}^{4} \gamma^{5}} \int_{-\infty}^{\infty} d \bar{\chi}\left|\int_{-\infty}^{\infty} \tau \exp \left\{i f\left[\left(1+\bar{\chi}^{2}\right) \tau+\frac{1}{3} \tau^{3}\right]\right\} d \tau\right|^{2} \\
& \frac{d P_{\|}}{d \omega}=\frac{e^{2} \omega^{2}\left[\left(r_{B}+\rho\right) \Omega_{0}^{2}+r_{B} \omega_{B}^{2}\right]}{4 \pi^{2} c^{5} Q_{2}^{2} \gamma^{5}} \int_{-\infty}^{\infty} \bar{\chi}^{2}\left|\int_{-\infty}^{\infty} \tau \exp \left\{i f\left[\left(1+\bar{\chi}^{2}\right) \tau+\frac{1}{3} \tau^{3}\right]\right\} d \tau\right|^{2} d \bar{\chi} .
\end{aligned}
$$

and

After evaluating the above integrals (see Appendix B), we obtain

$$
\frac{d P_{\perp}}{d \omega}=-\frac{\sqrt{3} e^{2}\left[\left(r_{B}+\rho\right) \Omega_{0}^{2}+r_{B} \omega_{B}^{2}\right]^{2} \gamma}{4 \pi c^{4} Q_{2}^{2} r_{c}^{*}}\left\{\frac{\omega}{\omega_{c}}\left[K_{2 / 3}\left(\frac{\omega}{\omega_{c}}\right)+\int_{\omega / \omega_{c}}^{\infty} K_{5 / 3}(y) d y\right]\right\},
$$

where we have used $f=3 \omega /\left(4 \omega_{c}\right)=\omega /\left(2 \gamma^{3} Q_{2} c\right)$. Similarly, we obtain

$$
\frac{d P_{\|}}{d \omega}=-\frac{\sqrt{3}}{2} \frac{e^{2} \gamma}{2 \pi} \frac{1}{r_{c}^{*}}\left\{\frac{\omega}{\omega_{c}}\left[\int_{\omega / \omega_{c}}^{\infty} K_{5 / 3}(y) d y-K_{2 / 3}\left(\frac{\omega}{\omega_{c}}\right)\right]\right\} .
$$

The total radiation power spectrum is given by

$$
\begin{aligned}
\frac{d P}{d \omega} & =\frac{d P_{\|}}{d \omega}+\frac{d P_{\perp}}{d \omega} \\
& =-\frac{\sqrt{3} e^{2} \gamma \omega}{4 \pi r_{c}^{*} \omega_{c}}\left\{\left[\int_{\omega / \omega_{c}}^{\infty} K_{5 / 3}(y) d y-K_{2 / 3}\left(\frac{\omega}{\omega_{c}}\right)\right]+\frac{\left[\left(r_{B}+\rho\right) \Omega_{0}^{2}+r_{B} \omega_{B}^{2}\right]^{2}}{c^{4} Q_{2}^{2}}\left[\int_{\omega / \omega_{c}}^{\infty}+K_{5 / 3}(y) d y+K_{2 / 3}\left(\frac{\omega}{\omega_{c}}\right)\right]\right\},
\end{aligned}
$$

where $r_{c}^{*}=c^{2} /\left[\left(r_{B}+\rho\right) \Omega_{0}^{2}+r_{B} \omega_{B}^{2}\right]$. For the synchrotron radiation, $\Omega_{0}=0, Q_{2}=\left(\sin ^{2} \alpha\right) / r_{B}, r_{B}^{2} \omega_{B}^{2}=c^{2} \sin ^{2} \alpha$, we get $\left[\left(r_{B}+\rho\right) \Omega_{0}^{2}+r_{B} \omega_{B}^{2}\right]^{2} /\left(c^{4} Q_{2}^{2}\right)=1$ and

$$
\frac{d P}{d \omega}=-\frac{\sqrt{3} e^{2} \gamma \omega_{B} \sin \alpha}{2 \pi c} \frac{\omega}{\omega_{c}} \int_{\omega / \omega_{c}}^{\infty} K_{5 / 3}(y) d y
$$

For the curvature radiation, $r_{B}=0, \Omega_{0}=c / \rho$, and $Q_{2}=1 / \rho$, we find $\left[\left(r_{B}+\rho\right) \Omega_{0}^{2}+r_{B} \omega_{B}^{2}\right]^{2} /\left(c^{4} Q_{2}^{2}\right)=1$ again, and

$$
\frac{d P}{d \omega}=-\frac{\sqrt{3} e^{2} \gamma}{2 \pi \rho} \frac{\omega}{\omega_{c}} \int_{\omega / \omega_{c}}^{\infty} K_{5 / 3}(y) d y
$$

To find the total power of radiation, we integrate over all frequencies and obtain

$$
P=\frac{-3 \sqrt{3} e^{2} \gamma^{4} c Q_{2}}{8 \pi r_{c}} \llbracket\left\{\left(1+\frac{\left[\left(r_{B}+\rho\right) \Omega_{0}^{2}+r_{B} \omega_{B}^{2}\right]^{2}}{c^{4} Q_{2}^{2}}\right\} \Gamma\left(\frac{7}{3}\right) \Gamma\left(\frac{2}{3}\right)+\left\{\frac{\left[\left(r_{B}+\rho\right) \Omega_{0}^{2}+r_{B} \omega_{B}^{2}\right]^{2}}{c^{4} Q_{2}^{2}}-1\right\} \Gamma\left(\frac{4}{3}\right) \Gamma\left(\frac{2}{3}\right) \rrbracket .\right.
$$

From equation (3.35) we can easily calculate the total power of the synchrotron and curvature radiations [N.B., $\left.\Gamma(7 / 3) \Gamma(2 / 3)=8 \pi / 9\left(3^{1 / 2}\right)\right]$. For the synchrotron radiation, we have

$$
P_{\mathrm{syn}}=-\frac{2 e^{4} B^{2} \sin ^{2} \alpha \gamma^{2}}{3 m^{2} c^{3}}
$$

For the curvature radiation, we have

$$
P_{\mathrm{cur}}=-\frac{2}{3} \frac{e^{2} c \gamma^{4}}{\rho^{2}}
$$

\section{DISCUSSION}

We have derived a universal radiation formula for a charged particle moving in curved magnetic field lines with arbitrary pitch angle, which can reduce to the formula for either synchrotron radiation or curvature radiation in appropriate limits. Figures 1-5 show the comparison between synchro-curvature radiation (solid line), synchrotron radiation (dashed line), and curvature radiation (dotted line) for different parameters. We can see that the synchro-curvature curves overlap with either synchrotron curves for $\sin \alpha \gg r_{B} / \rho$ or curvature curves for $\sin \alpha \ll r_{B} / \rho$ as expected. However, they can differ from each other significantly when $\sin \alpha \approx r_{B} / \rho$. It is very important to note that there are regions in the pulsar magnetosphere where parameters of charged particles can vary considerably from place to place. For example, charged particles in the acceleration regions, i.e., polar gap (see Ruderman \& Sutherland 1975) or outer gap (Cheng, Ho, \& Ruderman 1986a, b), can start with a large pitch angle and evolve to a very tiny pitch angle. Neither the simple synchrotron radiation nor the curvature radiation theories can correctly describe this evolution of pitch angle, because they are only valid in the extreme limits of the pitch angle. 


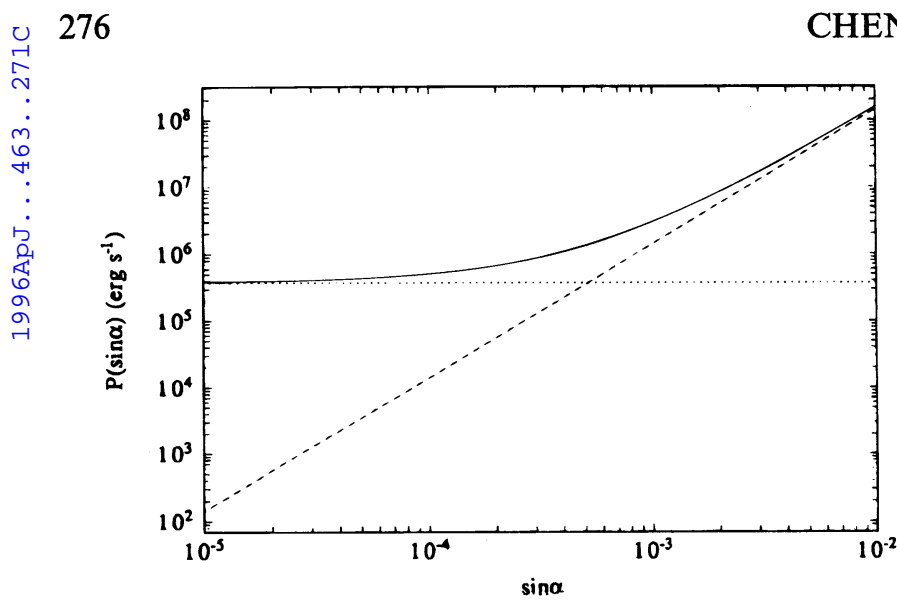

Fig. $1 a$

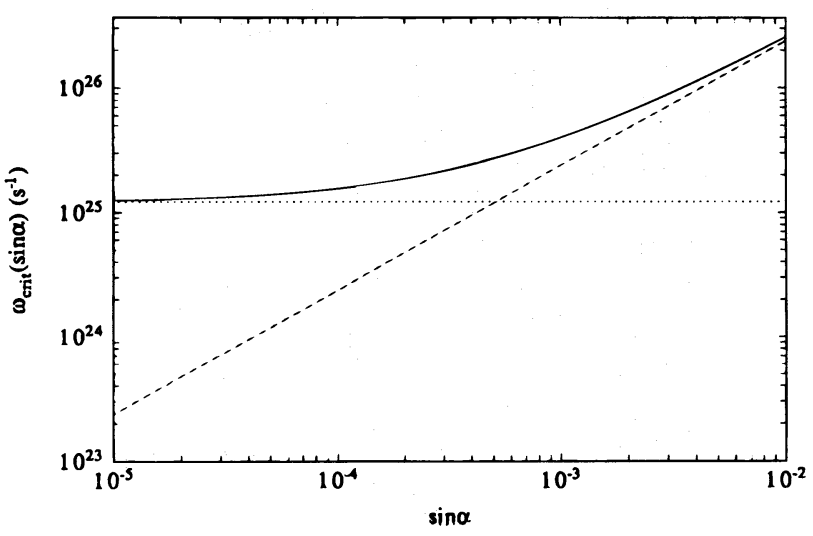

FiG. $1 b$

FIG. 1.-(a) Radiation power, $p$, and (b) characteristic frequency, $\omega_{\text {crit }}$, as a function of $\sin \alpha$ (for $\gamma=3 \times 10^{7}, \mathrm{~B}=10^{6} \mathrm{G}$, and $\rho=10^{8} \mathrm{~cm}$ ) for the synchro-curvature mechanism (solid curve), synchrotron mechanism (dashed line), and curvature mechanism (dotted line).

On the other hand, our formula can more consistently describe the radiation process for arbitrary pitch angle. Furthermore, the pitch angle determines the characteristic energy of the emitted photons, which eventually controls the potential of the gap via pair production (Sturrock 1971; Ruderman \& Sutherland 1975; Arons \& Scharleman 1979). This may also explain why the spectra of six known gamma-ray pulsars can differ so much even though their underlying radiation and pair production processes are so similar (Cheng \& Zhang 1995; Zhang \& Cheng 1995).

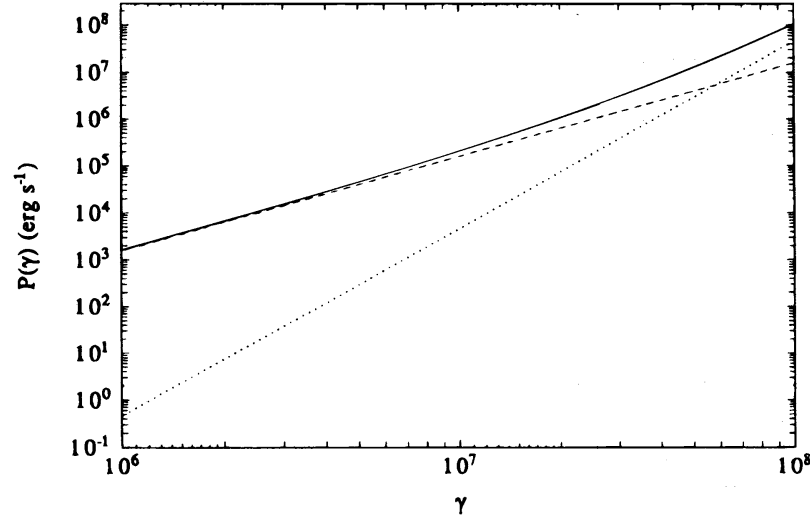

FIG. $2 a$

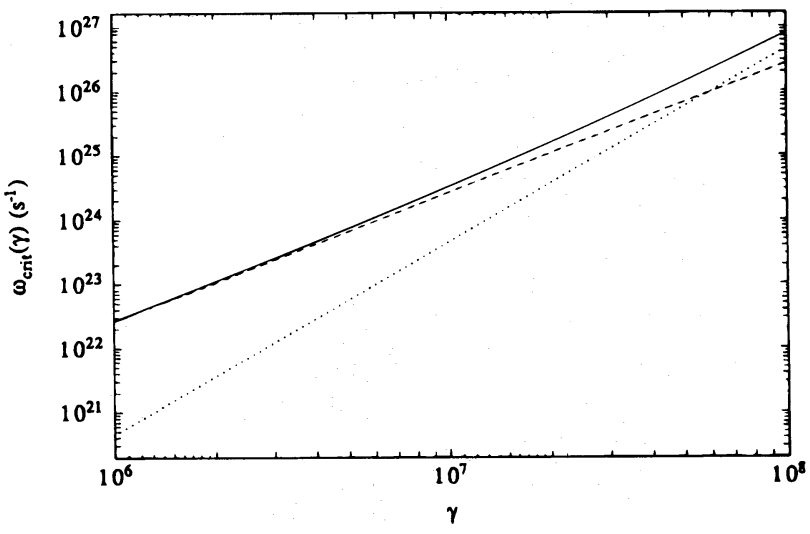

Fig. $2 b$

FIG. 2. - (a) $P$ and (b) $\omega_{\text {crit }}$ vs. $\gamma$ for $\sin \alpha=10^{-3}, \rho=10^{8} \mathrm{~cm}$, and $B=10^{6} \mathrm{G}$

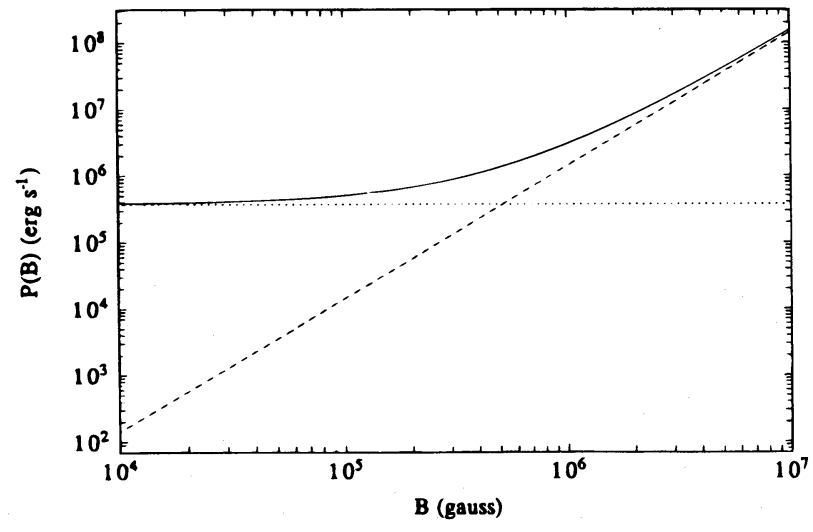

FIG. $3 a$

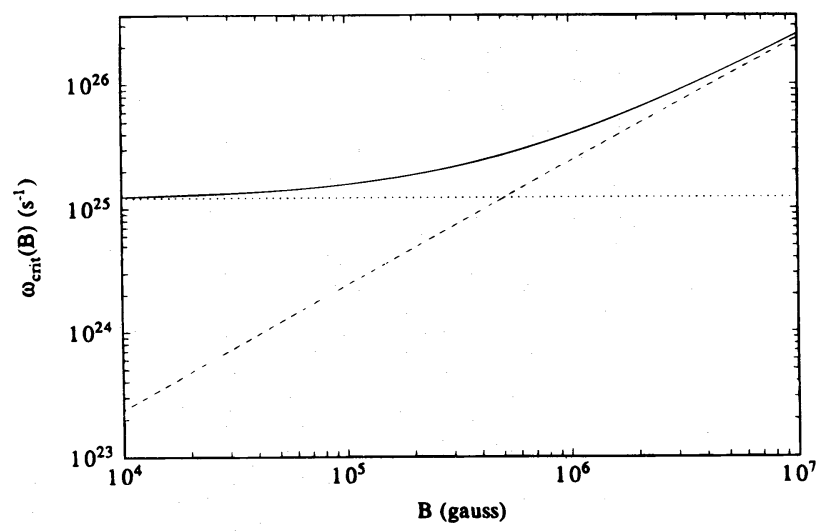

Fig. $3 b$

FIG. 3. $-(a) P$ and (b) $\omega_{\text {crit }}$ vs. $B$ for $\sin \alpha=10^{-3}, \rho=10^{8} \mathrm{~cm}$, and $\gamma=3 \times 10^{7}$ 


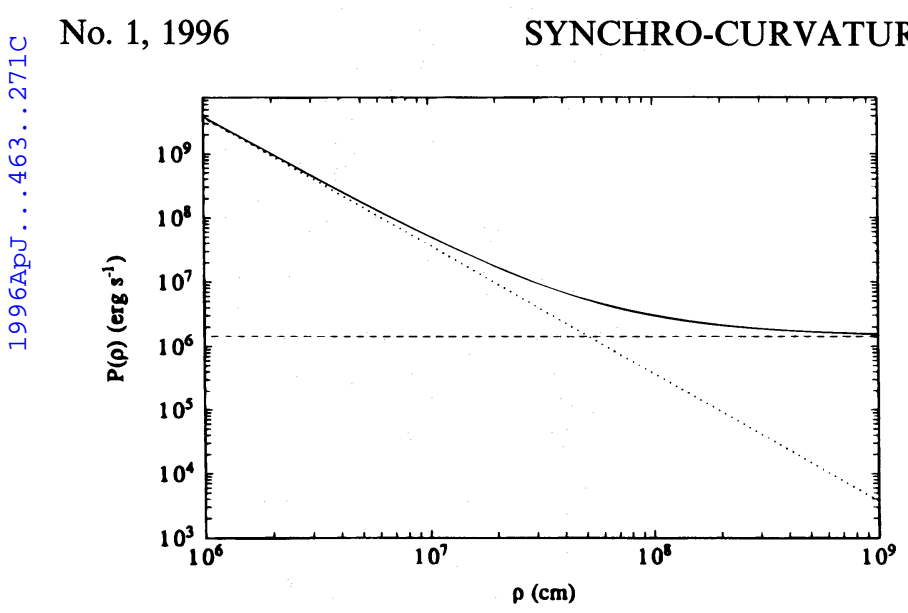

Fig. $4 a$

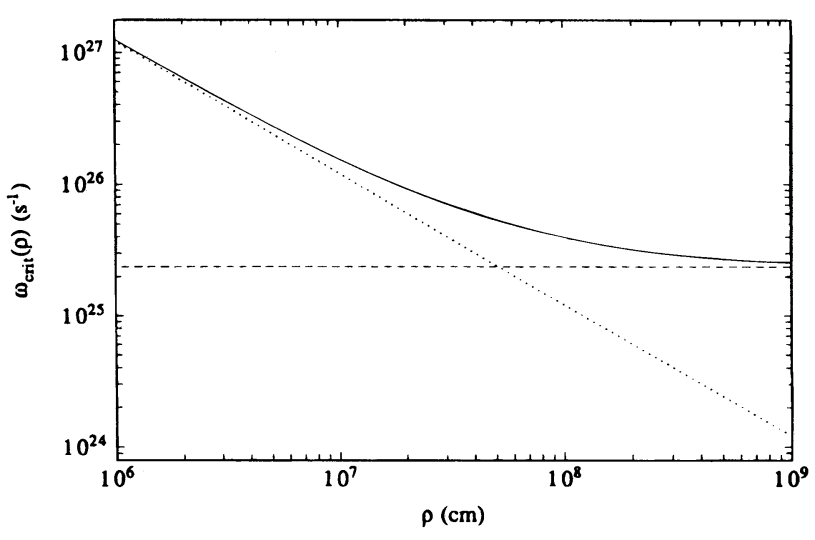

Fig. $4 b$

FIG. 4. $-(a)$ and $(b) \omega_{\text {crit }}$ vs. $\rho$ for $\sin \alpha=10^{-3}, \gamma=3 \times 10^{7}$, and $B=10^{6} \mathrm{G}$

The degree of polarization is defined as

$$
\pi(\omega)=\left(\frac{d P_{\perp}}{d \omega}-\frac{d P_{\|}}{d \omega}\right) /\left(\frac{d P_{\perp}}{d \omega}+\frac{d P_{\|}}{d \omega}\right)
$$

which can be an important quantity to differentiate the synchro-curvature radiation from both sychrotron radiation and curvature radiation, because the $K_{2 / 3}\left(v / v_{c}\right)$ component in the expressions $d P_{\perp} / d \omega$ and $d P_{\|} / d \omega$ is not completely canceled. The degree of polarization for synchrotron radiation and curvature radiation can be expressed as

$$
\pi(\omega)=\left[K_{2 / 3}\left(\frac{\omega}{\omega_{c}}\right) / \int_{\omega / \omega_{c}}^{\infty} K_{5 / 3}(y) d y\right],
$$

where $\omega_{c}$ for synchrotron radiation and curvature radiation is given by equations (3.11) and (3.12), respectively. The degree of polarization for synchro-curvature is

$$
\pi_{\mathrm{sc}}(\omega)=\frac{Q_{+} \pi(\omega)+Q_{-}}{Q_{-} \pi(\omega)+Q_{+}},
$$

where $Q_{+}=1 /\left(Q_{2} r_{c}^{*}\right)^{2}+1, Q_{-}=1 /\left(Q_{2} r_{c}^{*}\right)^{2}-1$, and $\omega_{c}$ is given by equation (3.10). In Figure 6 we compare the degree of polarization of these three mechanisms. At high frequencies, the polarization of all these mechanisms approaches unity. This is easily understood because $K_{2 / 3}\left(\omega / \omega_{c}\right)=\int_{\omega / \omega_{c}}^{\infty} K_{5 / 3}(y) d y$ for $\omega / \omega_{c} \gg 1$. At low frequencies, the polarization of synchrotron radiation and curvature radiation both appraoch $50 \%$, but the polarization of synchro-curvature radiation approaches $42 \%$, which is actually the numerical value of $\left[3-\left(Q_{2} r_{c}^{*}\right)^{2}\right] /\left[3+\left(Q_{2} r_{c}^{*}\right)^{2}\right]$. Again, this results from the fact that $2 K_{2 / 3}\left(\omega / \omega_{c}\right)=$ $\int_{\omega / \omega_{c}}^{\infty} K_{5 / 3}(y) d y$ for $\omega / \omega_{c} \ll 1$. Therefore, in principle we can differentiate the radiation resulting from each mechanism by measuring the degree of polarization.

This work is partially supported by grants from the UPGC of Hong Kong and the National Natural Science foundation of China. We thank T. C. Chan for numerical computations and preparation of the figures and Terry Boyce for a critical reading of our manuscript.

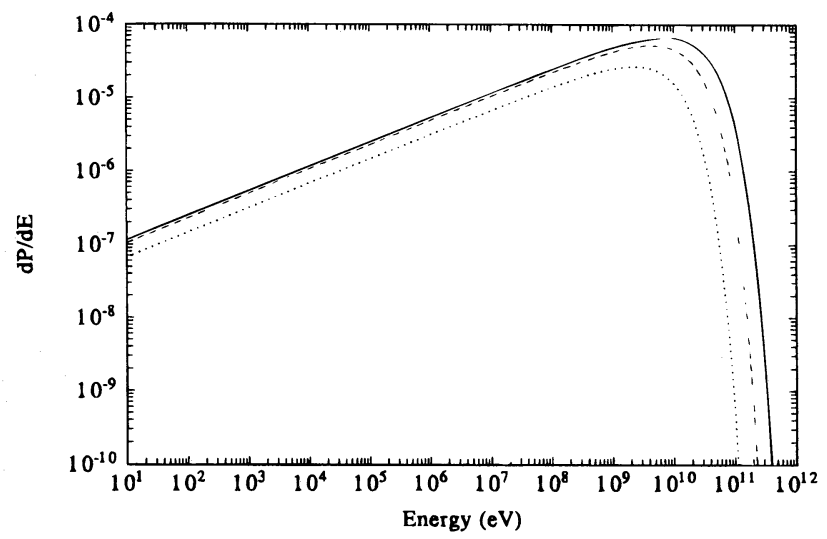

Fig. 5. - Power spectrum for $\sin \alpha=10^{-3}, \rho=10^{8} \mathrm{~cm}, \gamma=3 \times 10^{7}$, and $B=10^{6} \mathrm{G}$ 


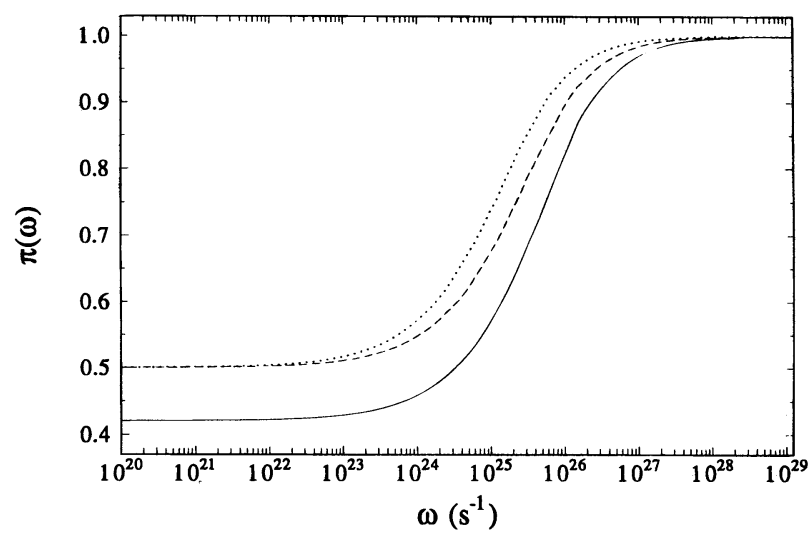

Fig. 6.-Comparison of the polarization from synchrotron radiation (dashed line), curvature radiation (dotted line), and synchro-curvature radiation (solid line). The parameters are the same as for Fig. 5 .

\section{APPENDIX A}

\section{EVALUATION OF SPECTRAL ENERGY DENSITY}

The spectral energy density for an arbitrary accelerated particle in the range of solid angle between $\Omega_{0}$ and $\Omega_{0}+d \Omega_{0}$, and in the frequency range between $\omega$ and $\omega+d \omega$ is given by (Peratt 1991)

$$
\frac{d^{2} E}{d \omega d \Omega}=\frac{e^{2} \omega^{2}}{4 \pi^{2} c}\left|\int_{-T / 2}^{T / 2} e^{i \omega[t-(n \cdot r) / c]}[n \times(n \times \beta)] d t\right|^{2} .
$$

To evaluate the above integral, we first note that

$$
\boldsymbol{n} \cdot \boldsymbol{r}=\rho \cos \theta_{0} \sin \Omega_{0} t+\frac{r_{B}}{2} \cos \theta_{0} \sin \left(\Omega_{0}+\omega_{0}\right) t+\frac{r_{B}}{2} \cos \theta_{0} \sin \left(\Omega_{0}-\omega_{0}\right) t+r_{B} \sin \theta_{0} \sin \omega_{B} t .
$$

Expanding the sine functions appearing in equation (A2) about $t=0$, we obtain

$$
\boldsymbol{n} \cdot \boldsymbol{r}=\left[\left(\rho+r_{B}\right) \Omega_{0} \cos \theta_{0}+r_{B} \omega_{B} \sin \theta_{0}\right] t-\left[\left(\rho+r_{B}\right) \Omega_{0}^{3} \cos \theta_{0}+3 \Omega_{0} \omega_{B}^{2} r_{B} \cos \theta_{0}+r_{B} \omega_{B}^{3} \sin \theta_{0}\right] \frac{t^{3}}{6} .
$$

From equation (2.2),

$$
\Omega_{0}=\frac{v_{\varphi}^{(G)}}{\rho}=\frac{v_{\varphi}}{\rho}=\frac{\gamma m v_{\varphi} v_{z} c}{e B_{0} \rho^{2}}
$$

and

$$
v_{\varphi}=v_{\|}=v \cos \alpha, \quad v_{z}=r_{B} \omega_{B} \cos \omega_{B} t=v \sin \alpha \cos \omega_{B} t,
$$

we can show that the average value of $v_{\varphi} v_{z}$ over a period should be zero, i.e.,

$$
\left\langle v_{\varphi} v_{z}\right\rangle_{T}=0 \text {. }
$$

Hence, on average, we have

$$
\Omega_{0}=\frac{v_{\varphi}}{\rho}=\frac{v_{\|}}{\rho}=\frac{v \cos \alpha}{\rho},
$$

where $\alpha$ is the inclination angle between $\boldsymbol{v}$ and $\boldsymbol{B}$. Therefore,

$$
\frac{\boldsymbol{n} \cdot \boldsymbol{r}}{c}=\left[\cos \left(\alpha-\theta_{0}\right)+\frac{r_{B}}{\rho} \cos \alpha \cos \theta_{0}\right] \frac{v}{c} t-\left[\frac{\left(\rho+r_{B}\right)}{\rho^{3}} \cos ^{3} \alpha \cos \theta_{0}+\frac{3}{\rho r_{B}} \cos \alpha \sin ^{2} \alpha \cos \theta_{0}+\frac{1}{r_{B}^{2}} \sin \theta_{0} \sin ^{3} \alpha\right] \frac{v^{3}}{c} \frac{t^{3}}{6} \text {. }
$$

The factor in equation (A1) can be expressed as

$$
e^{i \omega[t-(\boldsymbol{n} \cdot \boldsymbol{r}) / c]}=\exp \left[i f\left(Q_{1} \tau+\frac{1}{3} \tau^{3}\right)\right],
$$


where

$$
\begin{gathered}
Q_{1}^{2} \equiv 1-2 \gamma^{2}\left[2 \sin ^{2}\left(\frac{\alpha+\theta_{0}}{2}\right)-\frac{r_{B}}{\rho} \cos \alpha \cos \theta_{0}\right], \\
Q_{2}^{2} \equiv\left(\frac{r_{B}^{2}+\rho r_{B}-3 \rho^{2}}{\rho^{3}} \cos ^{3} \alpha \cos \theta_{0}+\frac{3}{\rho} \cos \alpha \cos \theta_{0}+\frac{1}{r_{B}} \sin ^{3} \alpha \sin \theta_{0}\right) \frac{1}{r_{B}}, \\
\tau \equiv c Q_{2} \gamma t, \\
f \equiv \frac{1}{2} \frac{\omega}{\gamma^{3} Q_{2} c},
\end{gathered}
$$

and when the integration variable is changed from $t$ to $\tau \propto \gamma t$, we can extend the upper and lower limits of the integral in equation (A1) to $\pm \infty$, because $\gamma$ is large enough. As a result, $\pm \gamma T / 2$ is changed to $\pm \infty$. Next we consider

$$
\begin{aligned}
\boldsymbol{n} \times(\boldsymbol{n} \times \boldsymbol{v}) & =\left(-\cos \theta_{0} \boldsymbol{i}^{0}+\sin \theta_{0} \boldsymbol{k}^{0}\right) \times(\boldsymbol{n} \times \boldsymbol{v}) \\
& =-\left(v_{z} \sin \theta_{0} \cos \theta_{0}+v_{x} \sin ^{2} \theta_{0}\right) \boldsymbol{i}^{0}-v_{y} \boldsymbol{j}^{0}-\left(v_{z} \cos ^{2} \theta_{0}+v_{x} \sin \theta_{0} \cos \theta_{0}\right) \boldsymbol{k}^{0} .
\end{aligned}
$$

In order to consider the polarization of the radiation simultaneously, we use a coordinate plane $X^{\prime \prime}, Y^{\prime \prime}$ which is perpendicular to the direction of $\boldsymbol{n}$. Also we assume that $\hat{\boldsymbol{y}}^{\prime \prime} \| \hat{\boldsymbol{y}}$, and express the inclination angle between $\hat{\boldsymbol{x}}^{\prime \prime}$ and $\hat{\boldsymbol{x}}$ as $\pi / 2-\theta_{0}$. Then the unit vector $\hat{\boldsymbol{x}}^{\prime \prime}$ in the $x^{\prime \prime}$-direction can be expressed as

$$
\begin{aligned}
\hat{\boldsymbol{x}}^{\prime \prime} & =\cos \left(\frac{\pi}{2}-\theta_{0}\right) \boldsymbol{i}^{0}+\cos \theta_{0} \boldsymbol{k}^{0} \\
& =\sin \theta_{0} \boldsymbol{i}^{0}+\cos \theta_{0} \boldsymbol{k}^{0} .
\end{aligned}
$$

We have

$$
\begin{aligned}
\boldsymbol{n} \times(\boldsymbol{n} \times \boldsymbol{v}) & =-\left(v_{z} \cos \theta_{0}+v_{x} \sin \theta_{0}\right)\left(\sin \theta_{0} \boldsymbol{i}^{0}+\cos \theta_{0} \boldsymbol{k}^{0}\right)-v_{y} \boldsymbol{j}^{0} \\
& =-\left(v_{z} \cos \theta_{0}+v_{x} \sin \theta_{0}\right) \hat{\boldsymbol{x}}^{\prime \prime}-v_{y} \hat{\boldsymbol{y}}^{\prime \prime}
\end{aligned}
$$

By putting the values for $v_{x}, v_{y}$ and $v_{z}$ calculated from equation (2.7) into the above equation, we have

$$
\{\boldsymbol{n} \times \boldsymbol{n}(\boldsymbol{n} \times \boldsymbol{v})\}_{\boldsymbol{x}^{\prime \prime}}=\left(\rho+r_{\boldsymbol{B}}\right) \Omega_{0} \sin \theta_{0}-r_{\boldsymbol{B}} \omega_{B} \cos \theta_{0}
$$

and

$$
\{\boldsymbol{n} \times(\boldsymbol{n} \times \boldsymbol{v})\}_{\boldsymbol{y}^{\prime \prime}}=\left[\left(\rho+r_{B}\right) \Omega_{0}^{2}+r_{B} \omega_{B}^{2}\right] t .
$$

In both equations we have used $\sin \Omega_{0} t \approx \Omega_{0} t$, noting that $t \equiv\left[1 /\left(c Q_{2} \gamma\right)\right] \tau$. From equation (A1), we obtain

$$
\frac{d^{2} E_{\perp}}{d \omega d \Omega}=\frac{e^{2} \omega^{2} A_{\perp}^{2}}{4 \pi^{2} c}\left|\int_{-\infty}^{\infty} \tau \exp \left[i f\left(Q_{1} \tau+\frac{1}{3} \tau^{3}\right)\right] d \tau\right|^{2},
$$

where

$$
A_{\perp}^{2} \equiv\left[\left(\rho+r_{B}\right) \Omega_{0}^{2}+r_{B} \omega_{B}^{2}\right]^{2} \frac{1}{c^{6} Q_{2}^{4} \gamma^{4}}
$$

At the same time, we have

$$
\frac{d^{2} E_{\|}}{d \omega d \Omega}=\frac{e^{2} \omega^{2} A_{\|}^{2}}{4 \pi^{2} c}\left|\int_{-\infty}^{\infty} \exp \left[i f\left(Q_{1} \tau+\frac{1}{3} \tau^{3}\right)\right] d \tau\right|^{2},
$$

where

$$
A_{\|}^{2} \equiv\left[\left(\rho+r_{B}\right) \Omega_{0}^{2} \sin \theta_{0}-r_{B} \omega_{B} \cos \theta_{0}\right]^{2} \frac{1}{c^{2} Q_{2}^{2} \gamma^{2}} .
$$

The integrals in equations (A16) and (A18) can be expressed in terms of the modified Bessel functions, i.e.,

$$
\begin{gathered}
\int_{-\infty}^{\infty} \tau \exp \left[i f\left(Q_{1} \tau+\frac{1}{3} \tau^{3}\right)\right] d \tau=\frac{2 Q_{1}^{2}}{\sqrt{3}} K_{2 / 3}(\eta), \\
\int_{-\infty}^{\infty} \exp \left[i f\left(Q_{1} \tau+\frac{1}{3} \tau^{3}\right)\right] d \tau=\frac{2 Q_{1}}{\sqrt{3}} K_{1 / 3}(\eta),
\end{gathered}
$$


where $\eta=\frac{2}{3} f$. Finally, we can calculate $\left(d^{2} E_{\perp} / d \omega d \Omega\right)$ and $\left(d^{2} E_{\|} / d \omega d \Omega\right)$ :

$$
\begin{aligned}
& \frac{d^{2} E_{\perp}}{d \omega d \Omega}=\frac{e^{2} \omega^{2} A_{\perp}^{2} Q_{1}^{4}}{3 \pi^{2} c} K_{2 / 3}^{2}(\eta), \\
& \frac{d^{2} F_{\|}}{d \omega d \Omega}=\frac{e^{2} \omega^{2} A_{\|}^{2} Q_{1}^{2}}{3 \pi^{2} c} K_{1 / 3}^{2}(\eta) .
\end{aligned}
$$

\section{APPENDIX B}

\section{EVALUATION OF THE POWER SPECTRUM}

In order to obtain an analytic expression for $d P / d \omega$, we should not express the square of the modulus of the integrals in equations (3.24) and (3.25) by using the modified Bessel functions. Let us rewrite the square of the modulus of the integrals as a product of two self-conjugate complex numbers, namely,

$$
\mid\left.\int_{-\infty}^{\infty} \tau \exp \left\{\text { if }\left[\left(1+\bar{\chi}^{2}\right) \tau+\frac{1}{3} \tau^{3}\right]\right\} d \tau\right|^{2}=\int_{-\infty}^{\infty} d \tau_{1} \int_{-\infty}^{\infty} \tau_{1} \tau_{2} \exp \left\{\text { if }\left(\tau_{1}-\tau_{2}\right)\left[\left(1+\bar{\chi}^{2}\right) \tau+\frac{1}{3}\left(\tau_{1}^{2}+\tau_{1} \tau_{2}+\tau_{2}^{2}\right)\right]\right\} d \tau_{2} .
$$

If we rotate the axis of integration variables by $\pi / 2$, i.e.,

$$
\xi \equiv \frac{1}{2}\left(\tau_{1}-\tau_{2}\right), \quad \zeta \equiv \frac{1}{2}\left(\tau_{1}+\tau_{2}\right),
$$

we have

$$
\tau_{1}^{2}+\tau_{1} \tau_{2}+\tau_{2}^{2}=\xi^{2}+3 \zeta^{2}
$$

and after the transformation of variables, the Jacobian becomes

$$
|J|=\frac{\partial\left(\tau_{1}, \tau_{2}\right)}{\partial(\xi, \zeta)}=\left|\begin{array}{rr}
1 & 1 \\
-1 & 1
\end{array}\right|=2
$$

Therefore,

$$
\begin{aligned}
\left|\int_{-\infty}^{\infty} \tau \exp \left\{i f\left[\left(1+\bar{\chi}^{2}\right) \tau+\frac{1}{3} \tau^{3}\right]\right\} d \tau\right|^{2} & =2 \int_{-\infty}^{\infty} d \xi \exp \left\{2 i f\left[\left(1+\bar{\chi}^{2}\right)+\frac{\zeta^{2}}{3}\right] \xi\right\} \int_{-\infty}^{\infty}\left(\zeta^{2}-\xi^{2}\right) \exp \left(2 i f \xi \zeta^{2}\right) d \zeta \\
& =2 \int_{-\infty}^{\infty}\left(\frac{\pi}{2 f|\xi|}\right)^{1 / 2} e^{i s \pi / 4}\left(-\xi^{2}+\frac{i}{4 f \xi}\right) \exp \left\{2 i f\left[\left(1+\bar{\chi}^{2}\right) \xi+\frac{\xi^{3}}{3}\right]\right\} d \xi,
\end{aligned}
$$

where $s=+1$ or -1 , depending on the sign of $\xi$. Using the same approach, we can have

$$
\left|\int_{-\infty}^{\infty} \bar{\chi} \exp \left\{i f\left[\left(1+\bar{\chi}^{2}\right) \tau+\frac{1}{3} \tau^{3}\right]\right\} d \tau\right|^{2}=2 \int_{-\infty}^{\infty} \bar{\chi}^{2}\left(\frac{\pi}{2 f|\xi|}\right)^{1 / 2} e^{i s \pi / 4} \exp \left\{2 i f\left[\left(1+\bar{\chi}^{2}\right) \xi+\frac{\xi^{3}}{3}\right]\right\} d \xi .
$$

Substituting this into equation (3.37), we obtain

$$
\begin{aligned}
\frac{d P_{\perp}}{d \omega} & =\frac{e^{2} \omega^{2}\left[\left(r_{B}+\rho\right) \Omega_{0}^{2}+r_{B} \omega_{B}^{2}\right]^{2}}{4 \pi^{2} c^{6} Q_{2}^{4} \gamma^{5} r_{c}} \int_{-\infty}^{\infty} d \bar{\chi}\left|\int_{-\infty}^{\infty} \tau \exp \left\{i f\left[\left(1+\bar{\chi}^{2}\right) \tau+\frac{1}{3} \tau^{3}\right]\right\} d \tau\right|^{2} \\
& =\frac{e^{2} \omega^{2}\left[\left(r_{B}+\rho\right) \Omega_{0}^{2}+r_{B} \omega_{B}^{2}\right]^{2}}{4 \pi^{2} c^{6} Q_{2}^{4} \gamma^{5} r_{c}^{*}} \int_{-\infty}^{\infty} d \xi\left(\frac{\pi}{2 f|\xi|}\right)^{1 / 2} e^{i s \pi / 4}\left(-\xi^{2}+\frac{i}{4 f \xi}\right) \int_{-\infty}^{\infty} \exp \left\{2 i f\left[\left(1+\bar{\chi}^{2}\right) \xi+\frac{\xi^{3}}{3}\right]\right\} d \bar{\chi} .
\end{aligned}
$$

But

$$
\int_{-\infty}^{\infty} \exp \left\{2 i f\left[\left(1-\bar{\chi}^{2}\right) \xi+\frac{\xi^{3}}{3}\right]\right\} d \bar{\chi}=\exp \left[2 i f\left(\xi+\frac{\xi^{3}}{3}\right)\right] \int_{-\infty}^{\infty} \exp \left(-2 i f \bar{\chi}^{2} \xi\right) d \bar{\chi}=\left[2 i f\left(\xi+\frac{\xi^{3}}{3}\right)\right] e^{i s \pi / 4} \frac{\sqrt{\pi}}{\sqrt{2 f|\xi|}}
$$

Therefore

$$
\begin{aligned}
\frac{d P_{\perp}}{d \omega} & =\frac{e^{2} \omega^{2}\left[\left(r_{B}+\rho\right) \Omega_{0}^{2}+r_{B} \omega_{B}^{2}\right]^{2}}{4 \pi^{2} c^{6} Q_{2}^{4} \gamma^{5} r_{c}^{*}} e^{i \pi / 2} \int_{-\infty}^{\infty} \frac{2 \pi}{2 f|\xi|}\left(-\xi^{2}+\frac{i}{4 f|\xi|}\right) \exp \left[2 i f\left(\xi+\frac{1}{3} \xi^{3}\right)\right] d \xi \\
& =\frac{e^{2} \omega^{2}\left[\left(r_{B}+\rho\right) \Omega_{0}^{2}+r_{B} \omega_{B}^{2}\right]^{2}}{2 \pi c^{6} Q_{2}^{4} \gamma^{5} r_{c}^{*}} \frac{i}{2 f}\left[-\int_{-\infty}^{\infty} \xi \exp \left[2 i f\left(\xi+\frac{1}{3} \xi^{3}\right)\right] d \xi+i \int_{-\infty}^{\infty} \frac{1}{4 f} \frac{1}{\xi^{2}} \exp \left[2 i f\left(\xi+\frac{1}{3} \xi^{3}\right)\right] d \xi\right]
\end{aligned}
$$


where the integral is given by

$$
\int_{-\infty}^{\infty} \frac{1}{\xi^{2}} \exp \left[2 i f\left(\xi+\frac{1}{3} \xi^{3}\right)\right] d \xi=-2 i f \int_{-\infty}^{\infty} \frac{1}{\xi} \exp \left[2 i f\left(\xi+\frac{1}{3} \xi^{3}\right)\right] d \xi-2 i f \int_{-\infty}^{\infty} \xi \exp \left[2 i f\left(\xi+\frac{1}{3} \xi^{3}\right)\right] d \xi .
$$

There are only two integrals we need to take care of, and they can be expressed as (Shu 1991)

$$
\begin{aligned}
& I_{1}=-2 i f \int_{-\infty}^{\infty} \xi \exp \left[2 i f\left(\xi+\frac{1}{3} \xi^{3}\right)\right] d \xi=\sqrt{3} G\left(\frac{4}{3} f\right), \\
& I_{2}=-2 i f \int_{-\infty}^{\infty} \frac{1}{\xi} \exp \left[2 i f\left(\xi+\frac{1}{3} \xi^{3}\right)\right] d \xi=-2 \sqrt{3} G\left(\frac{4}{3} f\right)+\sqrt{3} F\left(\frac{4}{3} f\right),
\end{aligned}
$$

where

$$
\begin{aligned}
& F(x)=x \int_{x}^{\infty} K_{5 / 3}(y) d y, \\
& G(x)=x K_{2 / 3}(x) .
\end{aligned}
$$

Therefore,

$$
\frac{d P_{\perp}}{d \omega}=-\frac{\sqrt{3} e^{2}\left[\left(r_{B}+\rho\right) \Omega_{0}^{2}+r_{B} \omega_{B}^{2}\right]^{2} \gamma}{4 \pi c^{4} Q_{2}^{2} r_{c}^{*}}\left\{\frac{\omega}{\omega_{c}}\left[K_{2 / 3}\left(\frac{\omega}{\omega_{c}}\right)+\int_{\omega / \omega_{c}}^{\infty} K_{5 / 3}(y) d y\right]\right\},
$$

where we have used $f=(3 \omega) /\left(4 \omega_{c}\right)=\omega /\left(2 \gamma^{3} Q_{2} c\right)$. Similarly, we obtain

$$
\frac{d P_{\|}}{d \omega}=-\frac{\sqrt{3}}{2} \frac{e^{2} \gamma}{2 \pi} \frac{1}{r_{c}^{*}}\left\{\frac{\omega}{\omega_{c}}\left[\int_{\omega / \omega_{c}}^{\infty} K_{5 / 3}(y) d y-K_{2 / 3}\left(\frac{\omega}{\omega_{c}}\right)\right]\right\} .
$$

From equations (B13) and (B14), we can obtain the total radiation power, i.e.,

$$
\begin{aligned}
\frac{d P}{d \omega} & =\frac{d P_{\|}}{d \omega}+\frac{d P_{\perp}}{d \omega} \\
& =-\frac{\sqrt{3} e^{2} \gamma \omega}{4 \pi r_{c}^{*} \omega_{c}}\left\{\left[\int_{\omega / \omega_{c}}^{\infty} K_{5 / 3}(y) d y-K_{2 / 3}\left(\frac{\omega}{\omega_{c}}\right)\right]+\frac{\left[\left(r_{B}+\rho\right) \Omega_{0}^{2}+r_{B} \omega_{B}^{2}\right]^{2}}{c^{4} Q_{2}^{2}}\left[\int_{\omega^{\prime} \omega_{c}}^{\infty} K_{5 / 3}(y) d y+K_{2 / 3}\left(\frac{\omega}{\omega_{c}}\right)\right]\right\} .
\end{aligned}
$$

\section{APPENDIX C}

\section{MODIFICATION OF THE RADIATION FORMULA CAUSED BY THE PARTICLE DRIFT}

We have shown that the drift velocity of a charged particle along the $z$-axis in constant circular magnetic lines is given by

$$
v_{z}^{(G)}=\frac{v^{2} \cos ^{2} \alpha}{\omega_{B} \rho}
$$

Thetotal velocity is actually given by

$$
v=v+v_{z}^{(G)}
$$

where $v$ is the particle velocity used in the main text, which has ignored the drift velocity of the guiding center. We can integrate the above equation and obtain

$$
r=r+v_{z}^{(G)} t
$$

where $\boldsymbol{r}$ is given by equation (2.7). It is straightforward to show that

$$
\boldsymbol{n} \cdot \boldsymbol{r}=\left[\left(\rho+r_{B}\right) \Omega_{0} \cos \theta_{0}+r_{B} \omega_{B} \sin \theta_{0}+v_{z}^{G} \cos \theta_{0}\right] t-\left[\left(\rho+r_{B}\right) \Omega_{0}^{3} \cos \theta_{0}+3 \Omega_{0} \omega_{B}^{2} r_{B} \cos \theta_{0}+r_{B} \omega_{B}^{3} \sin \theta_{0}\right] \frac{t^{3}}{6}
$$

and

$$
\boldsymbol{n} \cdot \boldsymbol{v}=v\left\{1-\left[2 \sin ^{2}\left(\frac{\alpha-\theta_{0}}{2}\right)-\frac{\Omega_{0}}{\omega_{B}} \sin \alpha \cos \theta_{0}-\frac{v_{e}^{(G)}}{v} \sin \theta_{0}\right]\right\}
$$


Therefore, the angle between $\boldsymbol{n}$ and $v^{\prime \prime}$ satisfies

$$
\cos \chi^{\prime}=\frac{v}{v^{\prime}}\left\{1-\left[2 \sin ^{2}\left(\frac{\alpha-\theta_{0}}{2}\right)-\frac{\Omega_{0}}{\omega_{B}} \sin \alpha \cos \theta_{0}-\frac{v_{z}^{(\mathbf{G})}}{v} \sin \theta_{0}\right]\right\} .
$$

Since $v \approx v^{\prime} \approx c$, we obtain

$$
x^{\prime 2}=2\left[2 \sin ^{2}\left(\frac{\alpha-\theta_{0}}{2}\right)-\frac{r_{B}}{\rho} \cos \alpha \cos \theta_{0}-\frac{v_{z}^{(G)}}{v} \sin \theta_{0}\right] .
$$

Now we can express the phase factor as

$$
i \omega\left(t-\frac{n \cdot r}{c}\right)=\frac{i \omega}{2 \gamma^{2}}\left(Q_{1}^{\prime 2} t+\frac{c^{2} Q_{2}^{2} \gamma^{2} t^{3}}{3}\right),
$$

where $Q_{1}^{\prime 2}=1+\gamma^{2} \chi^{\prime 2}$ and $Q_{2}^{2}$ is defined in equation (3.6). We can rewrite the phase factor as

$$
e^{i \omega\left[t-\left(n \cdot r^{\prime}\right) / c\right]}=\exp \left\{\text { if }\left[\left(1+\gamma^{2} \chi^{2}\right) \tau+\frac{1}{3} \tau^{3}\right]\right\},
$$

where $f$ is still defined by equation (3.7). The expression of the phase factor has a similar form as before, except that $\chi$ is replaced by $\chi^{\prime}$. Furthermore,

$$
\boldsymbol{n} \times \boldsymbol{n} \times \boldsymbol{v}^{\prime}=-\left[\left(v_{z}+v_{z}^{(G)}\right) \sin \theta_{0} \cos \theta_{0}+v_{x} \sin ^{2} \theta_{0}\right] \boldsymbol{i}^{0}-v_{y} \boldsymbol{j}^{0}-\left[\left(v_{z}+v_{z}^{(G)}\right) \cos ^{2} \theta_{0}+v_{x} \sin \theta_{0} \cos \theta_{0}\right] \boldsymbol{k}^{0} .
$$

The perpendicular and the parallel components are given by

$$
n \times\left(n \times v^{\prime}\right)_{x^{\prime \prime}}=\left(\rho+r_{B}\right) \Omega_{0} \sin \theta_{0}-\left(r_{B} \omega_{B}+\frac{v^{2} \cos ^{2} \alpha}{\omega_{B} \rho}\right) \cos \theta_{0}
$$

and

$$
n \times(n \times v)_{y^{\prime \prime}}=-v_{y},
$$

respectively. Using equation (C6), we can show that

$$
\left(\rho+r_{B}\right) \Omega_{0} \sin \theta_{0}-\left(r_{B} \omega_{B}+v_{z}^{(G)}\right) \cos \theta_{0}=c \sin \chi^{\prime} \approx c \chi^{\prime} .
$$

Then we get

$$
n \times(n \times v)_{\hat{x}^{\prime \prime}}=c \chi^{\prime} .
$$

We can see that the expressions for the two polarized spectral energy densities remain unchanged

$$
\frac{d^{2} E_{\|}}{d \omega d \Omega}=\frac{e^{2} \omega^{2}}{4 \pi^{2} c^{3} Q_{2}^{2} \gamma^{4}}\left|\int_{-\infty}^{\infty} \gamma \chi \exp \left\{i f\left[\left(1+\gamma^{2} x^{\prime 2}\right) \tau+\frac{1}{3} \tau^{3}\right]\right\} d \tau\right|^{2}
$$

and

$$
\frac{d^{2} E_{\perp}}{d \omega d \Omega}=\frac{e^{2} \omega^{2}\left[\left(r_{B}+\rho\right) \Omega_{0}^{2}+r_{B} \omega_{B}^{2}\right]^{2}}{4 \pi^{2} c^{7} Q_{2}^{4} \gamma^{4}}\left|\int_{-\infty}^{\infty} \tau \exp \left\{i f\left[\left(1+\gamma^{2} \chi^{\prime 2}\right) \tau+\frac{1}{3} \tau^{3}\right]\right\} d \tau\right|^{2}
$$

except $\chi$ is replaced by $\chi^{\prime}$. Again, the direction of the strongest radiation is $\chi^{\prime} \approx 0$, when we have

$$
\left(\rho+r_{B}\right) \Omega_{0} \sin \theta_{0}=\left(r_{B} \omega_{B}+v_{z}^{(G)}\right) \cos \theta_{0}
$$

or

$$
\cot \theta_{0}=\frac{\left(\rho+r_{B}\right) \Omega_{0}}{r_{B} \omega_{B}+v_{z}^{(G)}} .
$$

Since the drift velocity of the guiding center can be approximated by

$$
v_{z}^{(G)}=\frac{v^{2} \cos ^{2} \alpha}{\omega_{B} \rho}=\frac{\Omega_{0}}{\omega_{B}} \frac{v}{c} \cos \alpha \approx \frac{\Omega_{0}}{\omega_{B}} \cos \alpha,
$$

we have

$$
\tan \theta_{0}=\tan \alpha+\frac{\Omega_{0}}{\omega_{B}} .
$$

From this we can see that $\theta_{0}$ is not equal to $\alpha$ when the drift velocity is included. However, the correction is the very small term $\Omega_{0} / \omega_{B}$. This also causes a small correction to the characteristic frequency, which is given by

$$
\omega_{c}=\frac{3}{2} \gamma^{3} c \frac{1}{\rho}\left(\frac{r_{B}^{3}+\rho r_{B}^{2}-3 r_{B} \rho^{2}}{\rho r_{B}^{2}} \cos ^{3} \alpha \cos \theta_{0}+\frac{3 \rho}{r_{B}} \cos \alpha \cos \theta_{0}+\frac{\rho^{2}}{r_{B}^{2}} \sin ^{3} \alpha \sin \theta_{0}\right)^{1 / 2} .
$$


Now $\theta_{0} \approx \alpha+\Omega_{0} / \omega_{B}$ for a relativistic charged particle. Therefore, this change only causes a minor modification to the it characteristic frequency, the radiation spectrum, and the power loss, but the forms of these quantities remain unchanged.

101

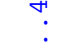

Arons, J., \& Scharleman, E. T. 1979, ApJ, 231, 854

Chang, D. B., \& Davis, L., Jr. 1962, ApJ, 136, 567

'á Cheng, K. S., Ho, C., \& Ruderman, M. A. 1986a, ApJ, 300, 500

II $1986 \mathrm{~b}, \mathrm{ApJ}, 300,522$

Cheng, K. S., \& Zhang, J. L. 1995, in preparation

Field, G. B. 1961, J. Geophys. Res., 66, 1395

Korchak, A. A. 1962, AZh, 40, 994

Mészáros, P. 1992, High-Energy Radiation from Magnetized Neutron

Stars (Chicago: Univ. Chicago Press)

Michel, F. C. 1991, Theory of Neutron Star Magnetospheres (Chicago:

REFERENCES

Univ. Chicago Press)

Ochelkov, Yu P., \& Usov, V. V. 1980, ApSS, 69, 439

Ortwein, N. R., Chang, D. B., \& Davis, L., Jr. 1966, ApJS, 12, 323

Peratto, A. L. 1991, Physics of the Plasma Universe (New York: Springer)

Ruderman, M. A., \& Sutherland, P. 1975, ApJ, 196, 57

Shu, F. H. 1991, The Physics of Astrophysics, Vol. 1 (Mill Valley: University Science Books)

Sturrock, P. 1971, ApJ, 164, 529

Thorne, K. S. 1963, ApJS, 8,

Zhang, J. L., \& Cheng, K. S. 1995, Phys. Lett. A, 208, 47 